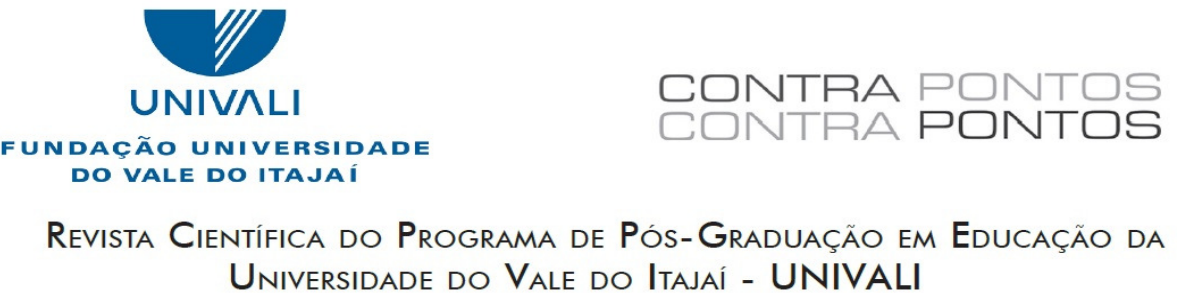

Volume 17 - N. 03 - Jul/Set. 2017

REITOR

Prof. Dr. Mário César dos Santos Vice-Reitora de GRAduAÇão

Profa ${ }^{a}$. Dra ${ }^{a}$. Cássia Ferri

Vice-Reitor de PesQuisa, Pós-Graduação,

Extensão e Cultura

Prof. Dr. Valdir Cechinel Filho

Vice-Reitor de Planejamento e

Desenvolvimento InSTITUCIONAL

Prof. Dr. Carlos Alberto Tomelin Procuradora Geral

Francieli Cristina Tirelli Pereira

DiRETOR ADMINISTRATIVo

Renato Osvaldo Bretzke, MSc.

\section{COMISSÃo EdTTORIAL}

Valéria Silva Ferreira

Verônica Gesser

Tânia Regina Raitz

Adair de Aguiar Neitzel

RESPONSÁVEL TÉCNICA

Mariana Soares da Silva

A qualidade e revisão das imagens dos artigos são responsabilidades dos seus autores

RESPONSÁvel

Francine Lucatelli

\section{Assistente de MARKeting Editora}

Daniele Torres Almeida

$$
\text { REVISÃo }
$$

Profa. Ana Cláudia Reiser de Melo, MSc

\section{Produção GráfICA}

Carolina Santana

LIVRARIA UNIVERSITÁRIA

Amanda Helena de Barros

Carina Gabrich Fernandes

$$
\text { TRADUÇÃo }
$$

Profa $^{a}$. Fiona Oliver Robson, MSc. Profa . Cynthia C. E. Philipps, MSc. Prof. Daniel Paglialunga, MSc.

$$
\text { (NELLE) - Espanhol }
$$

\section{Conselho Editorial Nacional}

Dr. Alfredo José Veiga Neto

Universidade Federal do Rio Grande do Sul (UFRGS)

Dr. Antonio Joaquim Severino

Universidade de São Paulo (USP)

Dra. Bernardete Angelina Gatti

Fundação Carlos Chagas (FCC)

Dra. Diana Carvalho Carvalho

Universidade Federal de Santa Catarina (UFSC)

Dra. Diva Spezia Ranghetti

Centro Universitário Católica de Santa Catarina (CATOLICA SC)

Dra. Doris Pires Vargas Bolzan

Universidade Federal de Santa Maria (UFSM)

Dr. Jadir de Morais Pessoa

Universidade Federal de Goiás (UFG)

Dr. Jefferson Mainardes

Universidade Estadual de Ponta Grossa (UEPG)

Dr. Lucidio Bianchetti

Universidade Federal de Santa Catarina (UFSC)

Dra. Maria Clara Bueno Fischer

Universidade Federal do Rio Grande do Sul (UFRGS)

Dra. Marilene Proença Rebello de Souza

Universidade de São Paulo USP

Dra. Roberta Gurgel Azzi

Universidade Estadual de Campinas (UNICAMP)

Dr. Roberto Antonio Deitos

Universidade Estadual do Oeste do Paraná (UNIOESTE)

Dr. Rogério da Silva Lima

Universidade de Brasília (UNB)

Dra. Terezinha Oliveira

Universidade Estadual de Maringá (UEM)

C768 Contrapontos. [recurso eletrônico]. - Vol. 17, n. 3 (abr./jun. 2017) - [Itajaí] : Editora da Universidade do Vale do Itajaí, 2017-

652 p. : fig. ; tab. ; qua.

Quadrimestral

No início a publicação era semestral

A revista tem acesso livre no Portal de Periódicos da UNIVALI

(www.univali.br/periódicos)

Resumo em português, inglês e espanhol.

Inclui referências.

Editores: Valéria Silva Ferreira, Verônica Gesser, Tânia Regina

Raitz e Adair de Aguiar Neitzel.

Revista Científica do Programa de Pós-Graduação em Educação

da Universidade do Vale do Itajaí.

ISSN: $1984-7114$

1. Educação - Periódicos. I. Revista Científica do Mestrado em Educação da Universidade do Vale do Itajaí. II. Título.

\section{CONSELHO EdITORIAL INTERNACIONAL}

Dra. Anne Kupiec

Université Paris Diderot (PARIS 7), Centre de Sociologie des Pratiques et des

Représentations Politiques, Paris, França Dr. José Augusto Pacheco

Universidade do Minho, Instituto de Educação, Braga, Portugal

Dra. Lilia Di Bello

Barry University, Education and Research Department, Miami, FL, Estados Unidos da América do Norte

Dra. Maria Pilar Figuera Gazo

Universitat de Barcelona (UB), Facultat d'Educació, Barcelona „Espanha

Dra. Patricia Friedrich

Arizona State University, School of

Humanities, Arts, and Cultural Studies, Phoenix, AZ, Estados Unidos da América do Norte

Dra. Cristina Mello

Universidade de Coimbra, Departamento de Línguas, Literaturas e Culturas, Coimbra, Portugal

Dr. Jonathan Tudge

University of North Carolina (UNC), Human

Development and Family Studies,

Greensboro, NC, Estados Unidos da America

\section{PARECERISTAS AD HOC DESTA EDIÇÃO}

Ademir Damazio (UNESC)

Adir Casaro Nascimento (UCDB)

Alboni Marisa Dudeque Pianovski Vieira (PUC/PR)

Ana Cristina Coll Delgado (UFPel)

Antonio Germano Magalhães Junior (UECE)

Cláudio José Oliveira (UNISC)

Evaldo Luis Pauly (UNILASALLE)

Genylton Odilon Rego da Rocha (UFPA) Gildo Volpato (UNESC)

Irene Jeanete Lemos Gilberto (UNIOESTE)

Marcelo de Andrade Pereira (UFMS)

Marcelo Silva de Souza Ribeiro (UNIVASF)

Maria Cleci Ventunini (UNICENTRO)

Maristani Polidori Zamperetti (UFPel)

Miguel Alfredo Orth (UFPel)

Vera Lúcia Chacon Valença (UNISUL) 